\title{
LA PROTESTA EN ESPAÑA Y BRASIL: CRISIS ECONÓMICA Y CRISIS POLÍTICA ${ }^{1}$ THE PROTEST IN SPAIN AND BRAZIL: ECONOMIC CRISIS AND POLITICAL CRISIS
}

\author{
Clemente Penalva Verdú \\ Departamento de Sociología II \\ Universidad de Alicante, España \\ Clemente.penalva@ua.es \\ Ana Targina Rodrigues Ferraz \\ Departamento de Serviço Social da \\ Universidade Federal do Espírito Santo, Brasil \\ anatargina@uol.com.br
}

\begin{abstract}
Resumen
El presente estudio es producto de la reflexión desarrollada por los autores a partir de a) la observación de las manifestaciones del Movimiento 15M y de las Jornadas de Junho en España y Brasil, respectivamente y b) el análisis de fuentes secundarias procedentes de estadísticas y de mecanismos de comunicación y difusión de actividades (websites y mass media). El principal objetivo del estudio es realizar un análisis comparativo entre los dos movimientos, revisando similaridades y diferencias en relación a los contextos de origen y sus desarrollos. Aunque se observan diferencias entre ambas protestas en cuanto a la coyuntura económica y política, así como al detonante de las protestas, si atendemos a los repertorios, contenidos y marcos de interpretación, se podrían observar ambas protestas como manifestaciones de un ciclo de protesta global que tiene sus raíces en la actual fase del capitalismo internacional.

Palabras clave: Movimientos sociales; acción colectiva; crisis capitalista global; Jornadas de Junho; 15M
\end{abstract}

\footnotetext{
${ }^{1}$ Este trabajo es producto del proyecto de investigación apoyad por European Commission FP7-PEOPLE-2012-IRSES. CIVIL-SW. Civil Engagement in Social Work: Developing Global Models. Project Coordinated by University of Coventry.
} 


\begin{abstract}
This study is the product of reflection developed by the authors from a) observation of the manifestations of 15M Movement and Jornadas de Junho in Spain and Brazil, respectively b) analysis of secondary sources from statistics and communication mechanisms and dissemination of activities (websites and mass media). The main objective of the study is a comparative analysis between the two movements reviewing similarities and differences in relation to the contexts of origin and their developments. Although differences between the two protests in regard to the economic and political situation, as well as differences in the trigger for the protests are observed, if we look at the repertoire, contents and frameworks of interpretation, one could observe both protests as manifestations of a cycle of global protest rooted in the current phase of international capitalism.
\end{abstract}

\title{
Extended abstract
}

What follows below is a comparative study of social protest in Spain (20112014) and Brazil (2013). Here is presented as two forms of political mobilization emerged in their civil societies that can represent respectively the tensions between State and Civil Society. Two groups of countries: Northern countries experiencing a bankruptcy of their Welfare State caused by the crisis; and emerging countries where after a period of growth and decline of social inequalities arise emerging social groups demanding greater participation in representative political system.

The growing process of cultural uniformity and development of communications makes societies to be interconnected in terms of knowledge and information. That is why the year 2011 marks the beginning of a cycle of protests worldwide that had a certain contagion effect (it was made it easier by the international media coverage) from the mass mobilizations initiated in Northern societies Africa and were spreading up in many countries. From this point of view, the mobilization in Spain had the closest reference from the "Arab Spring" mobilizations and the Brazilian was partially inspired by the Spanish and other mobilizations that took place in the years 2011 and 2012 elsewhere in the world. Although with significant differences due to different local and economic context, it is observed that the complaint of the effects of neoliberalism on local societies and on their social protection systems is both in $15 \mathrm{M}$ and Jornadas de Junho. These differences may explain the different motivation for the outbreak of popular mobilization. While in Spain protest is associated with a clear deterioration of living conditions; in Brazil, protest occurs in the context of increased improving expectations of them.

Some indicators that express these differences lead us to see from the comparative point of view, different hypotheses concerning the conditions of collective action. While the Spanish movement is a reaction to the disastrous end of the ascending cycle of consumption and upward social mobility; the Brazilian mobilization might result from the mismatch between the partial improvement of living conditions and lack of opportunities for political participation. 
The protests are reactions of both civil societies in front of the resignation of state to social protection and to improve living conditions.

We believe that deprivation and frustration of expectations are not enough to explain both mobilizations and we have to attend to other constraints (frames of interpretation and political opportunities), but it is noteworthy that in this case occurs a match between subjective and objective factors. This study shows how the objective data of socioeconomic status (deterioration in Spain and failure of expectations of improvement in Brazil) are complemented by the subjective perception of intergenerational social mobility. Thus, in terms of expectations of intergenerational social mobility in Spain a remarkable pessimism is perceived, while in Brazil is observed optimism.

From the point of view of the "frameworks of interpretation", the idea of "injustice" is contemplated in both scenarios and also the idea of political renewal and social substantially affecting the political structures of the state. These frameworks can provide to the protest the diagnosis, affective and cognitive development, as well as identifying aspects of belonging to the group and definition of political opponents. From this perspective, in both processes are detected the political opportunities arising from the perception of decomposing political systems, with a significant loss of legitimacy of institutions; and a political system away from the consensus reached after democratic transitions from dictatorships. It should be noted the coincidence between mobilizations in Spain in an election year (local and general) and the protests in Brazil occurred in the previous year for presidential and state elections; mobilizations that reflected dissatisfaction with the social democratic governments (PSOE and PT, respectively). Furthermore, in the Brazilian case, the protest coincided with international display of preparations for the World Cup the following year.

The cycles of protests come to express the process of experimentation, extension and intensification that occurs both spatially and along time. The concept is understood as a process that by prolonging the protest, causes the apparition and development of new organizations, strengthens the old and allows interaction between the new and the old, creating a kind of fusion which gives rise to a new framework of shared interpretation. In Spain, it is very interesting how come to converge in many protests persons (and sometimes groups) experienced in long struggles for social transformation with disenchanted people who are new to unconventional political participation and who are searching a series of reforms that will lead to the situation prior to the crisis. In Brazil, the mobilizations of June 2013 following the trail of struggles waged for over than a decade by the same MPL, with attempts at convergence and adaptation to the urban space of the ways of struggle of MST, and with the same anti-capitalist character.

In terms of political structure, the interpretation framework created is the decomposition of the old and building something new. The importance of interpretation frames has been emphasized by many authors, noting this important cognitive component and ideological at the same time of definition of the situation that compels individuals to collective action. These frames are conditioned 
by the cultural and historical tradition of collective action and, in turn, determine the "repertoire" of collective action that takes place at a certain time. Repertoires that intensify inherited search strategies of spectacular nature and symbolism, disobedience and peaceful occupation of public spaces that stimulate media coverage; repertoires that intensify both inherited search strategies of spectacular nature and symbolism (batucadas, carnival atmosphere, emotional expressiveness), disobedience and peaceful occupation of public spaces that stimulate media coverage; as new ones, closely related to the use of social media and technology have led to the production of numerous essays from an ethnographic perspective in connection with the emotional side or have applied to study protests from Spain and Brazil new concepts such as "techno-political" and the "cyberactivism". These studies include the ability of social networks (in real time, with the use of images and textual rhetoric of limited characters) to increase emotional identification with the movement both in times of outbreak as of development.

The study of social movements allows us to observe what is changing in a society in terms of mentality, customs, and political, economic and social practices. Also internationally. The movement started in the Arab Spring passed through the Iberian Peninsula to reach London and New York, Istanbul, Sao Paulo, Mexico City, Santiago de Chile, Hong Kong. With the process of economic and cultural globalization, movements have never been able to be addressed regardless of their interconnection, their mutual influence in the international arena. In a way, with the technological developments of communication is attending a more intense dialogue between movements in the past, and with their national specificities, you could even speak of a coral protest against capitalism as a form of social organization currently affecting all mankind.

\section{INTRODUCCIÓN}

En España no hay manifestación popular de protesta de cierta entidad que no cuente con una batucada (que expresa, entre otras cosas, ambiente festivo, multiculturalismo -de origen africano pero popularizado en Brasil- y acciones pacíficas). La forma (y sus "repertorios", Tarrow, 2004) de la protesta tiene significado, en este caso heredado de los movimientos altermundistas de finales de los años 90 y los 2000 que iniciaron un ciclo de protesta, marcado por el activismo global (Della Porta y Tarrow, 2005), que es anterior al que comienza en 2011, en el cual se integran las protestas que en este artículo tratamos. En cuanto al contenido reivindicativo y su evolución, tanto en Brasil como en España, los movimientos de protesta iniciados por el 15M (España) y los movimientos Jornadas de Junho o Movemento Passe Livre (Brasil) han desembocado en la creación de plataformas reivindicativas que reclaman profundos cambios políticos y sociales. En cuanto a las estructuras políticas, en España con el "Proceso Constituyente" diferentes colectivos de la izquierda política pretendían impul- 
sar un cambio que superara lo que se ha considerado un cierre en falso de la transición de la dictadura a la democracia. Un proceso que no alteró sustancialmente las estructuras de poder (ejército y fuerzas de seguridad, clases dominantes y propietarias, justicia, Iglesia, monarquía). En Brasil, las propuestas de reforma política tienen defensores a la derecha y a la izquierda políticas, con objetivos muy diferentes: restringir la participación para la derecha; y ampliarla para la izquierda.

Todas estas reinvindicaciones presentan una notable convergencia en repertorios y contenidos, pero difieren en la coyuntura económica y social. Mientras en España la protesta expresa una resistencia al cambio después de la caída estrepitosa desde el punto más alto del ciclo expansivo que otorgó una gran capacidad de consumo -y endeudamiento- a la población; la protesta en Brasil se produce en un momento de supuesta disminución de las desigualdades y de crecimiento de las clases medias (o mejora de las condiciones de la clase trabajadora) con una caída del número de brasileños y brasileñas pobres (de más de 61 millones a menos de 40 millones entre 2001 y 2009, lo que constituye un $21,4 \%$ de la población brasileña ${ }^{2}$ ) en función del programa de transferencia monetaria Bolsa Família, un programa de carácter focalizado (Clementi e Schettino, 2013).

En cierto modo son dos estímulos diferentes de movilización frente a la limitación del sistema político por parte de las dos sociedades civiles: resistencia y retorno a la situación anterior de las clases medias deterioradas en la sociedad española; y mejora de los servicios públicos (transporte, pero también educación y sanidad) con el incremento de expectativas de integración en el sistema político (y en la sociedad de consumo) de una clase trabajadora en proceso de transformación, en el caso de Brasil.

\section{METODOLOGÍA}

En este trabajo, se intenta encajar una serie de rasgos definitorios de las masivas protestas sociales de ambos países dentro de los conceptos y elaboraciones teóricas de la prolífica y diversa "teoría de los movimientos sociales". Por ello, a través de un análisis comparativo del contexto, los procesos y las formas de protesta de ambas series de acontecimientos se pretende dar un aporte sobre los condicionamientos estructurales y coyunturales de los conflictos políticos y las oportunidades políticas, así como los ciclos de protesta y el activismo global. Las fuentes de datos han sido muy diversas. Por un lado, datos secundarios proce-

${ }^{2}$ Según el último censo del IBGE (Instituto Brasileiro de Geografia e Estatística) (2010), Brasil tenía en 2010 190.755.799 habitantes. 
dentes de anuarios estadísticos de instituciones especializadas en la producción de datos socioeconómicos relativos a consumo y mercado de trabajo. Para los datos de Brasil se han consultado las bases de datos del Instituto Brasileiro de Geografia e Estatística (IBGE) y del DIEESE (Departamento Intersindical de Estatística e Estudos Socioeconômicos; para los datos relativos a España se han consultado los resúmenes estadísticos del INE (Instituto Nacional de Estadística). Otros datos incorporados en el estudio provienen del Banco Mundial (desigualdad a partir del índice Gini y uso de tecnología de comunicación). Los datos relativos a encuestas de opinión, de utilidad para contextualizar el clima político y la participación en la protesta se han utilizado datos del Centro de Investigaciones Sociológicas (CIS, España) y del diario Datafolha (Brasil).

Para la interpretación han sido muy útiles unas fuentes muy variadas de información procedente de medios de comunicación y de los órganos de difusión en Internet de los propios movimientos sociales implicados en las protestas. La consulta de estas fuentes no ha sido sistemática pero sí muy intensa, combinando la lectura de diferentes blogs y webs con la observación participante de los investigadores en diferentes asambleas y manifestaciones acontecidas en el periodo entre 2011 y 2014 en España, y entre 2013 y 2015 en Brasil. Un trabajo de campo poco planficado y estructurado pero que ha producido gran cantidad de información directa en los escenarios naturales donde se desarrollaban las acciones colectivas.

La combinación de diferentes métodos (documentación secundaria de datos e información publicada, así como la observación) ha posibilitado el análisis desde diferentes perspectivas (cuantitativa y cualitativa) la protesta dentro de su contexto económico, social y político.

\section{RESULTADOS}

\subsection{La protesta política en España: crisis económica y crisis política}

La gran abundancia de análisis de todo tipo, desde la literatura de las ciencias sociales a los documentos generados por los propios protagonistas, hace que se pueda hablar de las narrativas del movimiento 15M. Esta abundancia de literatura (destacamos Taibo 2011, 2012; Castells 2012; Domenech, 2014) sobre el movimiento es un indicador del interés, acorde con el enorme impacto mediático, que para la comunidad científica tuvo esta serie de movilizaciones que se extendieron en sus primeras formas de protesta desde mayo a agosto de 2011.

El 15M es un movimiento surgido en mayo de 2011 en diferentes ciudades españolas, que se extendió por todo el Estado en unas semanas y que tuvo su forma de expresión política en la forma de participación en diferentes mani- 
festaciones más o menos multitudinarias y en la ocupación de espacios públicos en los centros de las ciudades. Cientos de miles de ciudadanos participaron en las manifestaciones y un gran número de ellos "tomaron" las plazas en acampadas que se extendieron (en más de 60 españolas y en 15 ciudades del extranjero) durante semanas, hasta que las autoridades decidieron enviar fuerzas de orden público para disolverlas pese a la voluntad de los participantes de continuar con la ocupación.

La situación económica internacional de crisis que comenzó en 2008 con la quiebra de instituciones financieras en Estados Unidos, arrastró en su caída a todo el sector financiero europeo. En España, el fin de la burbuja inmobiliaria y financiera condujo a un enorme endeudamiento de las clases medias y populares. Tras unos años de notable expansión económica y del consumo, la población se enfrentaba a un panorama económico de desempleo (cerca de 5 millones de desempleados, más del $20 \%$ de la población activa; y un desempleo juvenil que se aproximaba al 45\%). El fuerte desempleo llevó a la incapacidad de hacer frente a las enormes deudas hipotecarias contraídas durante el período de expansión. Las ejecuciones de las hipotecas por impagos condujo a un gran número de desahucios.

Los efectos de la crisis se empezaron a sentir de forma abrupta sobre la mayoría de la población. La sociedad española cayó desde una situación de opulencia con los mayores índices de consumo sobre todo tipo de artículos y servicios de consumo suntuario, a una realidad de carencia de recursos. El índice de pobreza en 2013 alcanzaba el 21\%, unos 8 millones de personas. Al mismo tiempo, se asiste en el transcurso de la crisis a un aumento de la adquisición de bienes de lujo por parte de las clases superiores.

Estas variables económicas han continuado la tendencia negativa hasta la actualidad (2014), estabilizándose en unos índices mínimos que ha llevado a los gobiernos a esperar una posible recuperación. La persistencia de la crisis y las recetas de política económica y social orientadas a la austeridad, explican la evolución de la serie de movimientos surgidos bajo la inspiración del 15M. No obstante, para la población juvenil, la ausencia de perspectivas de futuro relacionadas con la seguridad, la emancipación o la construcción de proyectos familiares ya estaban presentes desde antes del inicio de la protesta. La juventud española que accedía al mercado laboral se caracterizaba por la precariedad y los bajos salarios. Unas situaciones laborales caracterizadas por unos contratos de trabajo marcados por la inestabilidad y la movilidad geográfica.

En este contexto socioeconómico, el nombre mediático asignado a los protagonistas de las protestas, "los indignados", expresa claramente la idea de protesta contra la injusticia. Injusticia social por las medidas gubernamentales 
conducentes a salvar el sector financiero (origen y motor de los factores que provocaron a la crisis) con recursos públicos (privatización de los beneficios y socialización de las pérdidas); e injusticia respecto a la asimetría a la hora de realizar "sacrificios" entre las diferentes clases para superarla. Así, la percepción de maltrato de la población se explica por esta situación precaria, pero también por la gestión política de la crisis y por la extensión de la corrupción en todos los niveles de la administración. En plena crisis, sobre un modelo productivo basado casi exclusivamente en la construcción, la percepción de los ciudadanos unió lo político y lo económico, identificándolos con la corrupción: las comisiones y sobrecostes, y la financiación ilegal de partidos políticos, se vinculan con el despilfarro en obras e infraestructuras públicas.

Los sucesivos gobiernos centrales, autonómicos y locales crearon las condiciones para el crecimiento de la burbuja financiero-inmobiliaria (reforma de la ley del suelo y reforma del sector financiero -derecha PP) y no supieron prever su explosión y sus consecuencias (izquierda PSOE). Gran parte de los procesos abiertos contra los políticos y empresarios vinculados con casos de corrupción no comienzan, se interrumpen por determinadas estrategias procesuales o terminan en veredictos y sentencias muy blandas en comparación con las penas impuestas a delitos comunes y con el padecimiento de la población en la crisis. Todo ello lleva a la percepción de impunidad ante la ley.

Desde 2011 se extiende la percepción en España de que la corrupción alcanza todas las instituciones protagonistas del proceso de transición de la dictadura a la democracia representativa entre los años 75 a 85 del siglo pasado: La Corona, los partidos políticos y los sindicatos mayoritarios, las organizaciones empresariales y financieras, las instancias del gobierno en todos los niveles del Estado, el Parlamento, la Iglesia y la Justicia. El principal efecto es la idea generalizada de que todas ellas han perdido legitimidad democrática y capacidad de representación de los intereses de la ciudadanía. Como indicaba uno de los principales eslóganes empleados en las protestas del 15M: "no nos representan".

Al margen de las prácticas relacionadas con la corrupción, la indignación con la clase política también tiene su origen en la ausencia de autonomía frente a los mercados. Por presiones de la Troika (FMI, Banco Central y Eurogrupo) las políticas públicas apuntan directamente al debilitamiento del Estado de Bienestar.

La reacción de la sociedad española produjo en 2011 un interesante proceso de extensión de prácticas de resistencia que surgen a partir de las tensiones experimentadas entre el Estado y su sociedad civil. La crisis económicofinanciera constituye una fuente de stress al que el sistema político no sólo ha sido incapaz de dar respuesta sino que lo ha acentuado, creando las condicio- 
nes para una revitalización de la sociedad civil (Alaminos y Penalva 2013). Desde esta perspectiva, se observa un intento de recuperación de los aspectos esenciales de la democracia, en el sentido de recuperación de un conjunto de elementos esenciales de la política que se perciben como degenerados. Así, desde la explosión de indignación y protesta de 2011, los movimientos sociales intentan recuperar las prácticas y valores de una verdadera democracia: participación en debates públicos abiertos entre iguales y poder de decisión en las decisiones sobre aspectos que afectan a toda la sociedad. En términos de estructura interna de los movimientos, se han de señalar la horizontalidad, la descentralización y la práctica de la democracia directa. Así, se pasó de la idea del partido político (o sindicato) como expresión y representación de sus intereses y como dirección de la acción política; a la autogestión y autopresentación sin mediadores.

En términos genealógicos, encontramos un comienzo, el 15 de mayo de 2011, y una forma de convocatoria notablemente novedosa frente a otras movilizaciones anteriores en España: las redes sociales tecnológicas. En cuanto a la motivación explícita y concreta tenemos dos grupos reivindicativos en la red: "no les votes", "juventud sin futuro" que convergieron en la plataforma de Internet "Democracia Real Ya". El primero surgió a partir de la protesta por parte de partidarios del conocimiento libre por la aprobación de la denominada Ley Sinde (apellido de la ministra de cultura que redactó la ley) tras las revelaciones de wikileaks que mostraba las presiones norteamericanas (de los lobbies de su industria cultural) para este cambio legislativo dirigido a la ilegalización de los archivos compartidos en Internet. Bajo el nombre "no les votes" los activistas propugnaba orientar el voto hacia cualquier partido minoritario como protesta contra los partidos mayoritarios que habían firmado la ley. "Juventud sin futuro" tiene una extraordinaria similitud con el movimiento "Geração à Rasca" portugués (Baumgarten, 2013) y denunciaba la situación de precariedad de gran parte de la juventud española con el lema: "sin casa, sin trabajo, sin pensión, sin miedo".

Por otro lado, el fenómeno mediático actuó como fuente de inspiración y como retroalimentador del 15M. En la misma medida en que fueron representadas en la TV las movilizaciones, fue creciendo la afluencia de ciudadanos de muy diferente origen social y edad que se sumaron a las acampadas de las principales ciudades españolas, bien participando en las asambleas y grupos de trabajo, bien ofreciendo alimentos y objetos para ayudar a mantener la ocupación de estos lugares públicos. El movimiento 15M gozó de un importante apoyo popular también en la opinión pública. Un año más tarde, en 2012, una encuesta mostraba como el $65 \%$ de la población (un 10\% más que en 2011) veía al 
$15 \mathrm{M}$ como un "movimiento que pretende regenerar la democracia" (Metroscopia, citado en Alaminos y Penalva, 2013).

El hecho de que las causas fundamentales de la protesta no han desaparecido (crisis económica y política), sino que se ha agravado y debido al mayor conocimiento de las prácticas de corrupción legales e ilegales experimentadas antes y durante la crisis; la protesta no ha cesado. Sin embargo se ha ido transformando en sus formas.

En su desarrollo, el análisis de los repertorios de acción colectiva nos lleva al encuentro con dos tipos de protesta: a) un tipo de participación política que supone una continuación $15 \mathrm{M}$, con un mayor peso del componente expresivo, emocional de la acción política (como muestran algunos lemas de la protesta en sus inicios "No es una crisis, es una estafa", "lo llaman democracia y no lo es", "Gobierno del pueblo para el pueblo", "Otra forma de hacer política es posible", "Política = amantes de Dinero", "Yo no soy anti-sistema, el sistema es anti-yo", "si no nos dejan soñar, no les dejaremos dormir") que tienen como marco de producción de discursos y prácticas la idea de transformación social hacia otro modelo productivo y medioambiental, de representación política, de cambio cultural ; y b) un tipo de participación política (con un mayor peso del componente racional, cognitivo) que tiene como marco de producción de discursos y prácticas la resistencia (retorno, adaptación) frente a la mutación del Estado. Como ejemplos del primer modelo tendríamos las convocatorias de "Rodea el Congreso" (también conocida como "25S" que propuso una acción simbólica consistente en rodear el lugar donde se desarrollan las actividades de los denostados "representantes políticos"); las "marchas de la dignidad" (una serie de columnas -apoyadas por más de 100 colectivos- reivindicativas realizaban el camino a pie desde todos los diferentes extremos de la península para converger en la capital central Madrid en marzo de 2014) y "escraches" (protestas y concentraciones en los domicilios o trabajos de las personas -políticos básicamente- a las que se quiere denunciar); y las plataformas de ayuda a las víctimas de la crisis (PAH -Plataforma de Afectados por la Hipoteca- y su campaña de "Stop Deshaucios" consistente en acciones de desobediencia civil y de resistencia pasiva ante las ejecuciones de desahucios. Dentro del segundo se identifican las "mareas ciudadanas" que defienden los servicios públicos ("marea verde" de defensa de educación pública, "marea blanca" de defensa de la Sanidad pública, "marea negra" de defensa de los funcionarios públicos, etc.). Respectivamente, coincidirían con las "dos almas" (Taibo, 2011) presentes en el 15M, respectivamente, reformista y transformadora.

Junto con estos dos modelos de participación ha surgido recientemente una forma de participación política convencional por la vía electoral: el partido 
"Podemos". Se trata de una formación política surgida en 2014 a partir de un manifiesto en cuyo título que queda claro su voluntad de expresar electoralmente el "espíritu" del 15M: Mover ficha: convertir la indignación en cambio político. Con una estructura inicial descentralizada basada en grupos locales ("círculos") irrumpió en el panorama político representativo en ese mismo año con la obtención en las elecciones europeas de un $8 \%$ de los votos y 5 escaños. Aunque en sus principios y formas de actuación Podemos no renuncia a las formas de participación política no convencional, aprovecha el voto como forma de canalización de la indignación. En los momentos de finalización de este trabajo las encuestas preelectorales ${ }^{3}$ otorgan a este partido una estimación del voto entre el 20 y el $30 \%$, porcentaje similar al de los partidos mayoritarios que se han alternado en el gobierno desde los años 80: PSOE y PP.

\subsection{Las Jornadas de Junho en Brasil: orígenes y algunos desarrollos}

En Junio de 2013 las calles de la mayor ciudad de Brasil, São Paulo, fueron tomadas por jóvenes manifestantes movilizados por el Movimento Passe Livre (MPL), reivindicando una revocación del aumento de los billetes del transporte público en aquella capital. El MPL, formado mayoritariamente por estudiantes de secundaria y universitarios, levantó en diferentes capitales brasileñas, desde 2005, la bandera del Passe Livre para todos y de la mejora de los servicios de transporte. Entretanto, desde los años 1970, con el intenso proceso de urbanización, se incrementan los problemas relacionados con la movilidad urbana, el acceso al ocio y a los servicios de salud y educación de la población trabajadora y pobre.

Para el Movimento Passe Livre la movilidad urbana no sólo supone el derecho al transporte público, gratuito y de buena calidad, sino también el acceso a los recursos urbanos y el derecho al disfrute de los bienes y servicios de la ciudad.

El 17 de junio de 2013, el uso y abuso de la fuerza por parte de la policía militar (balas de goma, spray de pimienta, gas lacrimógeno y fuego real) contra algo más de 4000 manifestantes, transformó la Avenida Paulista, principal foco de las manifestaciones en la ciudad de São Paulo, en un campo de batalla. Las escenas se extendieron por las redes sociales e incluso los grandes medios dieron visibilidad a los acontecimientos, desencadenando una inmensa ola de protestas por todo el país en apoyo, en principio, de los manifestantes y contra la represión policial.

\footnotetext{
3 (Metroscopia, enero de 2015 en El País http://elpais.com/elpais/2015/01/10/media/14209 25889_312799.html; Celeste-TEL em El diario.es http://www.eldiario.es/politica/PP-levementeaspiraciones-electorales-consolida_0_352065037.html; Barómetro CIS de enero de 2015 CIS http://datos.cis.es/pdf/Es3050mar_A.pdf)
} 
El crecimiento de las manifestaciones tanto en el número de participantes como en su extensión por varias ciudades brasileñas, incluyó un gran número de otras reivindicaciones. Además de la protesta contra los caros y precarios servicios de transporte público, también se convirtieron en objetos de denuncia y protesta los servicios de educación y salud (insuficientes y de baja calidad), los prejuicios raciales, sexuales y de género, la violencia generalizada (especialmente de la policía contra los residentes en las zonas periféricas de las grandes ciudades), el desalojo de millares de familias por efecto de las obras de la Copa Mundial de Fútbol en $2014^{4}$, la ocupación de tierras indígenas para los agronegocios y la construcción de plantas hidroeléctricas y contra la corrupción.

Esta última reivindicación fue protagonizada, principalmente, por los manifestantes de las clases medias, estimulados por los grandes medios, para quienes la corrupción de los servidores públicos explica la escasa disponibilidad y la deficiente aplicación de recursos en las políticas y servicios estatales. Las demás reivindicaciones contaban con movimientos organizados desde décadas atrás, pero ninguno de ellos hasta ese momento (a excepción del Movimento dos Trabalhadores Rurais Sem Terra/MST), había sido capaz de reunir tantos manifestantes en tan poco tiempo y simultáneamente en diferentes zonas de del país.

El hecho de que las demandas de las protestas de junio de 2013 contaran con movimientos sociales organizados en todo o país desde hace décadas, señala una importante diferencia entre las Jornadas de Junho y el 15M y los demás movimientos en España. En Brasil no se pasó por la experiencia del Estado de Bienestar, ni siquiera en su versión liberal como la define Esping-Andersen (1991). La ausencia de derechos, el desempleo, los bajos salarios, el trabajo desprotegido, los servicios públicos precarios y la corrupción son característicos del Estado Brasileño. Esas deficiencias, a partir de las luchas contra la dictadura militar en las décadas de 1970 e 1980 y por la construcción de la ciudadanía en el país, impulsaron la organización de innumerables y vigorosos movimientos sociales. Las luchas sociales y el esfuerzo por construir un Estado de Bienestar en el país a partir de la nueva constitución de 1988, fueron derrotadas por la adhesión de las élites económicas de los gobiernos brasileños a los dictámenes del neoliberalismo y por la reiterada inserción subordinada y dependien-

${ }^{4}$ Los Comités Populares de la Copa organizados en las ciudades sede de la competición han sido la forma de organización y resistencia por parte de los residentes de las zonas afectadas por las obras y de denuncia de los numerosas violaciones de los derechos ciudadanos y a una vivienda digna cometido por el Estado. La realización de la Copa Confederaciones en junio de 2013 hizo posible que las información sobre los costos de las obras, el gasto público y las violaciones tuvieran una mayor visibilidad. Aumentó la insatisfacción popular las restricciones a la participación de la gente en los eventos, como el hecho de que la mayoría de los estadios se encontraran en zonas de difícil acceso y que las entradas fueran caras. 
te en la economía mundial, manteniendo y ampliando el empobrecimiento de la población y de un Estado al servicio de los intereses del capital financiero especulativo.

En este sentido, la violencia represora contra las manifestaciones populares es también característica de la cultura autoritaria del Estado brasileño) para reprimir una protesta legítima del movimiento de estudiantes y trabajadores usuarios del transportes ${ }^{5}$, hizo de chispa sobre el barril de pólvora que suponía la intensidad de las numerosas insatisfacciones acumuladas relacionadas con las respuestas del Estado a sus necesidades más básicas (trabajo, salud, vivienda, transporte). Sin embargo, también contribuyó a la presencia de gran cantidad de manifestantes de las clases medias con reivindicaciones no siempre progresistas o igualitarias como las relacionadas con la reducción de la edad penal, el mantenimiento del carácter investigativo del Ministerio Público y el endurecimiento de penas para los delitos de corrupción.

Aunque Brasil es un país formalmente democrático desde mediados de los años 80 , continúa siendo un país extremadamente desigual. Las desigualdades en la distribución de la riqueza no han sufrido alteraciones profundas. No se produjo un efectivo cambio en la distribución de los rendimientos entre los estratos más ricos y más pobres de la población (Clementi y Schettino, 2013). Las desigualdades son incluso más perversas porque coinciden con las desigualdades de raza o género. Los hombres negros y las mujeres negras constituyen el segmento más pobre de la población brasileña y, según los datos del IBGE (2010) y del DIEESE (2014), tienen una baja escolarización, están subempleados y reciben los salarios más bajos.

En la última década, los programas de transferencia de renta para los más pobres como el de la Bolsa Família, de inclusión en la educación superior privado y de los aumentos del salario mínimo, crearon una disminución de las desigualdades que se puede considerar como superficial, ya que los mecanismos de medición de la renta de los más ricos son defectuosos y el programa mencionado sólo aumenta la capacidad de consumo de los muy pobres (Clementi y Schettino, 2013). Mientras, se agotó el aumento de la capacidad de consumo de una importante proporción de la población a partir de programas de facilitación del acceso al crédito y del aumento del salario mínimo desde la llegada del Partido dos Trabalhadores (PT) al gobierno federal. El endeudamiento y el consecuente agotamiento de la capacidad de consumo, y la alta inflación coincidente con la imposibilidad de acceso a los servicios educativos y de salud privados

\footnotetext{
${ }^{5}$ Segun el diario Datafolha (junio, 2013), entre los participantes de las manifestaciones de São Paulo mayores de 14 años, el 51\% eran asalariados y el 9\% autónomos; el 22\% poseían un título de enseñanza media y el $77 \%$ uno de educación superior.
} 
de calidad, colocan en cuestión la supuesta existencia de un país menos desigual y más justo.

La gran crisis capitalista de 2008, y las medidas tomadas por el gobierno brasileño para evitar el contagio, estimularon el crecimiento económico por medio de la construcción de grandes obras viarias, de producción de energía y de proyectos inmobiliarios a partir del programa de viviendas Minha Casa, Minha Vida creado en 2009. Tales iniciativas alejó, temporalmente, el fantasma del desempleo, pero la masa salarial todavía es baja y el sector que más empleos produces es el de servicios (DIEESE, 2012). Además, muchas de esas obras (incluidas las de la Copa 2014) produjeron el desalojo de miles de familias y aumentaron la especulación inmobiliaria, inflando el precio de venta de los inmuebles y de los alquileres.

Muchas de las iniciativas fueron producto de de las articulaciones realizadas entre el Gobierno Federal y los sectores directamente interesados, especialmente las industrias de construcción civil y las grandes contratas, en una demostración de la permanencia del carácter patrimonialista y privatista del Estado brasileño, donde los detentores de capital y tierras circulan con desenvoltura por las altas instancias de decisión, influenciándolas, particularmente, por medio de la corrupción.

En este sentido, algunas de las manifestaciones antipartidarias de São Paulo y de otras ciudades que rechazaban la presencia de militantes de partidos de izquierda y del PT, y que expresaban un carácter eminentemente conservador, también reflejaban insatisfacción con la representación parlamentaria, con la ausencia sistemática de participación popular en las grandes decisiones del país y con el modelo jerarquizado y centralizado de organización de sindicatos y de otros movimientos sociales.

El deseo de una democracia de nuevo tipo, más horizontal e inclusiva, que privilegiara el debate público y la participación popular, tanto por su carácter colectivo como por su condición abierta y transparente, se intensificó. El mismo modelo de organización del MPL (inexistencia de dirección formal, asambleas abiertas y participación de cualquier interesado, círculos de discusión), así como la existencia de innumerables colectivos juveniles y otros ${ }^{6}$ también organizados de forma horizontal, descentralizada y que hacen un uso sistemático de los nuevos medios y de las redes sociales como forma de articulación y comunicación (Gohn, 2013), apuntan hacia un nuevo modelo de organizaciones que presentan otras formas de expresión política, particularmente la participación directa en procesos de decisión.

${ }^{6}$ La organización colectiva horizontal y descentralizada a través de foros y colectivos ha sido particularmente importante en la organización del movimiento negro, LGBT (Lesbianas, Gays, Bisexuales, Transgéneros y Transexuales) y feminista. 
Aún así, los partidos políticos no fueron descartados. Pequeños partidos de izquierda fueron importantes para impulsar la organización de los colectivos y para darles apoyo político y organizacional, contribuyendo tanto a la reflexión política como a la activación de apoyos mutuos entre sindicatos y movimientos sociales. Lo que se puso en cuestión por parte de los manifestantes fue un modelo centralizado, elitista y poco transparente de los procesos decisorios de las instancias democráticas, en las que el distanciamiento entre los parlamentarios electos y los intereses y demandas de los electores es casi total.

Por lo tanto, las manifestaciones también señalaron una insatisfacción profunda con los procesos electorales trasformados en espectáculos de alto coste y que colocan a los financiadores de las mismas como los grandes tomadores de decisiones, en la medida que son sus intereses los que son representados por los parlamentarios, facilitando la corrupción. La realización de un plebiscito popular en septiembre de 2014 que reivindicaba el establecimiento de una Asamblea Constituyente exclusiva para la definición del sistema político ${ }^{7}$ y el alto número de abstenciones y de votos blancos y nulos en la última elección presidencial $\left(2014^{8}\right)$ parecen confirmar esa insatisfacción.

Las respuestas gubernamentales a las jornadas de junio de 2013 fueron extremamente tímidas ${ }^{9}$ teniendo en cuenta la cantidad de problemas denunciados y ni siquiera abordaron las razones más profundas de la desigualdad extrema: la superexplotación de los trabajadores brasileños y la enorme transferencia de recursos públicos para financiar el capital financiero y el sector privado. Brasil tiene comprometido el $42,04 \%$ de su presupuesto fiscal para el pago de parte de los intereses y amortizaciones de sus deudas; mientras que apenas el $3,49 \%$ y el $4,11 \%$ de este presupuesto se destina a políticas de educación y salud, respectivamente (Auditoria Cidadã da Dívida, 2014).

El fenómeno de las Jornadas de Junho aún no ha sido suficientemente analizado en Brasil. Tras un periodo de mayor intensidad, entre junio y julio de

${ }^{7}$ Cerca de 100 organizaciones populares entre movimientos sociales y partidos políticos, organizaron un referéndum en Brasil, del 1 al 7 de septiembre, con la siguiente pregunta: ¿Está usted a favor de una Asamblea Constituyente exclusiva y soberana sobre el sistema político?

${ }^{8}$ En la primera ronda, de los 142.822 .046 electores que forman el censo electoral , un $19,39 \%$ no compareció en las urnas; y del total de votantes, el 3,84\% votaron en blanco y el $5,80 \%$ votaron nulo. En la segunda vuelta, un $21,10 \%$ no votó y entre los votantes, el 1,71\% votó en blanco y 4,63\% votó nulo (UOL, 2014).

${ }^{9}$ Las medidas se redujeron a la suspensión del incremento de tarifas del transporte público en todos los municipios donde hubo un aumento anterior, la introducción del programa Mais Médicos (programa de estímulo orientado a ajustar el número de médicos brasileños y extranjeros en las ciudades donde la relación médico-población, de acuerdo con los criterios la ONU, es muy baja) y la asignación del $75 \%$ de los royalties derivados del pré-sal para la política educativa. 
2013, fue posible notar cómo uno de sus desarrollos fue el aumento de la capacidad de articulación y movilización de movimientos sociales de larga existencia como los sindicatos, las asociaciones de vecinos y los movimientos de lucha por la vivienda ${ }^{10}$. También se puede destacar el incremento de las redes sociales y de expresiones artísticas como la música o el teatro para dar visibilidad a las reivindicaciones de los movimientos.

Otro desarrollo importante ha sido el incremento de la organización de grupos y foros de lucha por políticas públicas, como mecanismo que convergencia y fortalecimiento de colectivos que persiguen el reconocimiento y acceso a derechos individuales y sociales. Con todo, el saldo más importante parece haber sido la recuperación de las acciones directa de enfrentamiento como huelgas, marchas y ocupaciones que constituyen una forma de vocalizar demandas y proyectos.

Por otro lado, se ha hecho patente la crítica a la negociación en gabinetes, los acuerdos, las reuniones entre líderes de los movimientos y agentes estatales; en definitiva, una serie de prácticas que apuntan a un modelo de decisiones que excluye la participación de las mayorías y donde está ausente la transparencia. En Brasil, tras la constitución de 1988, la participación popular vía Consejos Gestores de Políticas Públicas ${ }^{11}$ se institucionalizó, estableciendo en estos espacios la negociación -entre organizaciones de la sociedad civil y representantes del Estado- como un mecanismo para la toma de decisiones, especialmente en el ámbito de las políticas sociales. Su desarrollo entre los años 1990 y 2010, entre avances y retrocesos, generó principalmente la contención de la capacidad de movilización y el disciplinamiento de los movimientos sociales y organizaciones de la sociedad civil participantes en estos espacios, dentro de un modelo de gestión compartida. El modelo de los consejos gestores, por tanto, fue señalados en las Jornadas de Junho como un modelo agotado que simplemente reproducía el modelo representativo parlamentario ${ }^{12}$.

${ }^{10}$ Fue notable la capacidad de organización y movilización del Movimiento de los Trabajadores Sin Techo (MTST), en São Paulo; especialmente mostrada durante la discusión y votación del nuevo plan general urbano de la ciudad, cuando el movimiento ocupó vacíos urbanos y la propia Cámara Municipal reivindicando una reforma urbana y más recursos estatales para viviendas asequibles.

${ }^{11}$ Conquista social que pugnaba no sólo por el restablecimiento de la democracia, sino también por su ampliación y por el reconocimiento de las organizaciones de la sociedad civil como interlocutoras y sujetos capaces de participar en la toma de decisiones Fueron pensados como espacios que posibilitaran un mayor control de los ciudadanos sobre las decisiones y acciones del Estado, como un mecanismo innovador de ampliación de la participación democrática. Para saber más sobre el asunto recomiendo la lectura de los siguientes autores brasileños: Dagnino (2004), Avritzer (2007), Tatagiba (2012) y Bravo (2012).

${ }^{12}$ Los Conselhos Gestores de Políticas Públicas fueron pensados como espacios que permitieran un mayor control de los ciudadanos sobre las decisiones y acciones del Estado; 
Algunos de los desarrollos más visibles en las Jornadas de Junho todavía no apuntan hacia la construcción de otro proyecto societario, capaz no sólo de plantear críticas, sino también de presentar una alternativa de superación del capitalismo en Brasil. Expresan, no obstante, una insatisfacción más profunda con las condiciones de vida de la mayoría de los brasileños y parecen constituir un terreno importante para la construcción de esta alternativa.

\section{DISCUSIÓN. ANÁLISIS COMPARATIVO}

Una vez expuestas narrativamente las movilizaciones, haciendo referencia tanto a las causas como al proceso, intentamos sintetizar las semejanzas y diferencias entre ambos movimientos a partir de algunos datos estadísticos. Previamente a esta sistematización comparativa, se deben resaltar dos elementos que marcan diferencias estructurales. Por un lado en torno a la cuestión temporal, el delay de dos años entre ambos movimientos que hace que uno de ellos tenga más recorrido (España). Por otro lado el distanciamiento sociocultural y socioeconómico que marca profundas diferencias entre ambas sociedades. Sin embargo, el creciente proceso de uniformización cultural y de desarrollo de las comunicaciones hace que las sociedades están más interconectadas en términos de conocimiento e información. Es por ello que el año 2011 marca el inicio de un ciclo de protestas a nivel mundial que, como hemos señalado en más arriba, supone un cierto efecto de contagio -facilitado por la cobertura mediática internacional- desde las movilizaciones masivas iniciadas en las sociedades del Norte de África y que se van extendiendo por gran cantidad de países. Desde este punto de vista, las movilización en España tenía como referente cercano las movilizaciones de la llamada "Primavera Árabe" y la brasileña se inspiró parcialmente en las españolas y otras movilizaciones que tuvieron lugar en los años 2011 y 2012 en otros lugares del mundo.

Aunque con diferencias marcadas por el diferente contexto local y coyuntural, se observa que la denuncia de los efectos del neoliberalismo sobre las sociedades locales y sus sistemas de protección social están tanto en el 15M como en las Jornadas de Junho. Estas diferencias pueden explicar la diferente motivación a la hora del estallido de la movilización popular. Mientras en España la protesta va asociada a un claro deterioro de las condiciones de vida; en Brasil la protesta se produce en el contexto de un incremento de las expectativas de la mejora de las mismas.

Algunos indicadores que expresan estas diferencias nos conducen, desde el punto de vista comparativo, a observar diferentes hipótesis con respecto a

constituyendo, por tanto, un mecanismo innovador de la democratización, proporcionando una expansión de la participación democrática 
las condiciones de la acción colectiva. Mientras que la movilización española es una reacción al estrepitoso fin del ciclo ascendente del consumo y de la movilidad social ascendente; la movilización brasileña podría ser resultado del desajuste entre la mejora parcial de las condiciones de vida y la ausencia de oportunidades de participación política, así como la demanda de una mayor responsabilidad de los agentes estatales en la gestión de los recursos públicos, en un contexto estructural de dificultades de acceso y de precariedad de los servicios básicos. En primer lugar, tenemos el gráfico comparativo del desempleo juvenil. Con tendencias opuestas en los dos países y un considerable salto en el incremento de la población desempleada en España. Este cruce en la evolución temporal también se ve en un indicador de la capacidad de consumo; el relativo al número de líneas celulares en cada uno de los países. Si bien se trata de un indicador que por sí solo no es suficiente para medir la multidimensionalidad del consumo, sí sirve para observar las tendencias en el momento puntual de estallido de la protesta social.

Figura 1. Desempleo juvenil (18-25 años), 2002-2013 (Brasil y España)

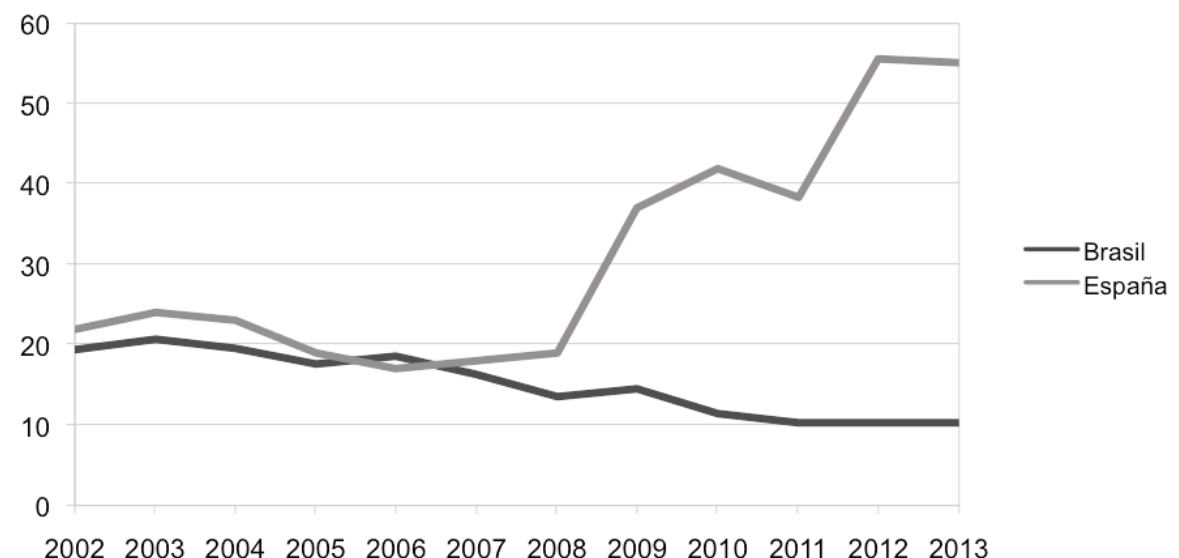

Fuente: elaboración propia a partir de los datos de INE (España) e IBGE (Brasil)

El consumo es un referente identitario de las clases medias. Ambos son movimientos de protesta urbanos con con un componente de clase media (emergente desde la mejora de las clases trabajadoras el caso brasileño, y en decadencia en el caso español), y espontáneos, a partir de unas convocatorias establecidas a partir de redes sociales tecnológicas que son accesibles desde estos dispositivos. El incremento de la capacidad de consumo sobre bienes que satisfacen un poco más allá de las necesidades básicas (como es el teléfono celular) muestran 
Figura 2. Abonos a teléfonos celulares cada 100 personas. 200-1013 (Brasil y España)

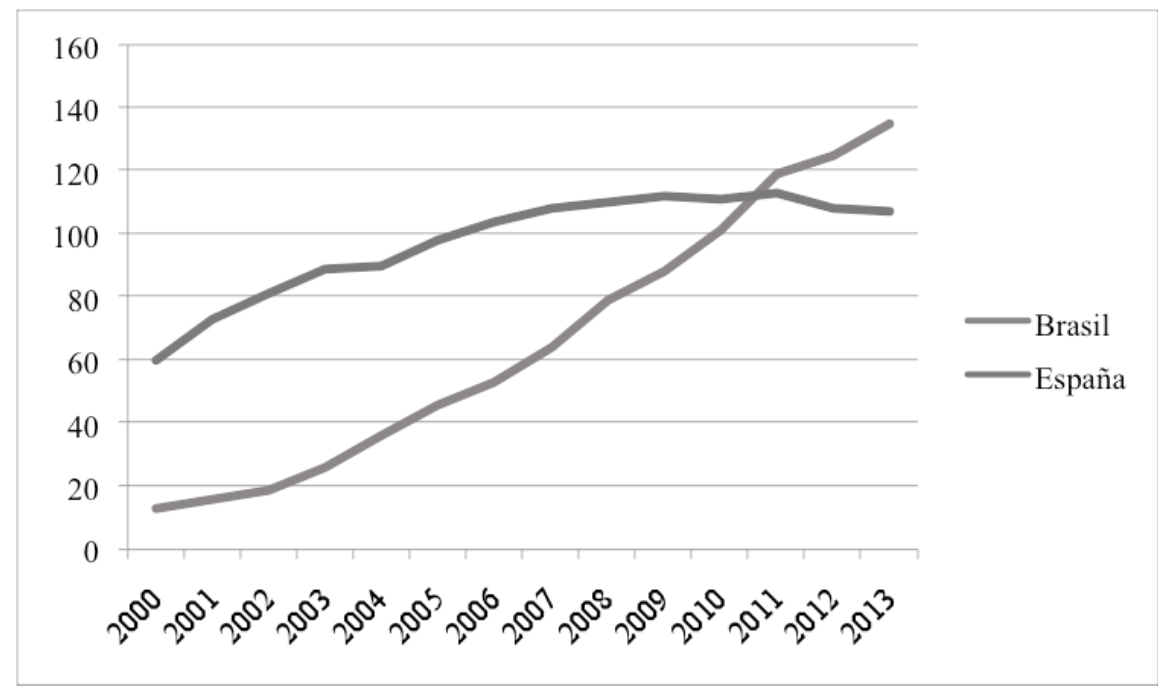

Fuente: elaboración propia a partir de Banco Mundial http://datos.bancomundial.org/ indicador/IT.CEL.SETS.P2

esta mejora de las clases trabajadoras, aunque las diferencias en torno a la desigualdad entre los dos países es patente (figura 3). Lo más importante aquí es observar el contraste entre la tendencia descendente en Brasil frente al pequeño incremento de la misma en España. No obstante, esta tendencia observada globalmente en el país Latinoamericano no permite ver las diferencias regionales (pensemos en la enorme extensión y heterogeneidad social) y hay que observar que Brasil ha sido durante muchos años uno de los países con mayor desigualdad del mundo.

Una síntesis de lo tratado anteriormente nos permite ver las diferencias y similitudes entre los dos movimientos a partir de una serie de elementos que tienen que ver con las formas (repertorios) de acción colectiva, las estructuras socioeconómicas, las oportunidades políticas (interacción con el sistema político), y la estructura y el proceso de los movimientos.

1) En cuanto a las reacciones del poder político, mientras en España éste se mantiene expectante en las primeras fases para desembocar en una disolución por vía de la represión; en Brasil es la represión primaria de gran violencia la que alimenta la protesta. La violencia, no obstante, tiene mayor cotidianidad en la sociedad brasileña, sobre todo en las zonas urbanas y contra la población más desfavorecida, y muy acorde con la violencia estructural de la desigualdad. 
Figura 3. Evolución del índice Gini (2001-2012) en Brasil y España

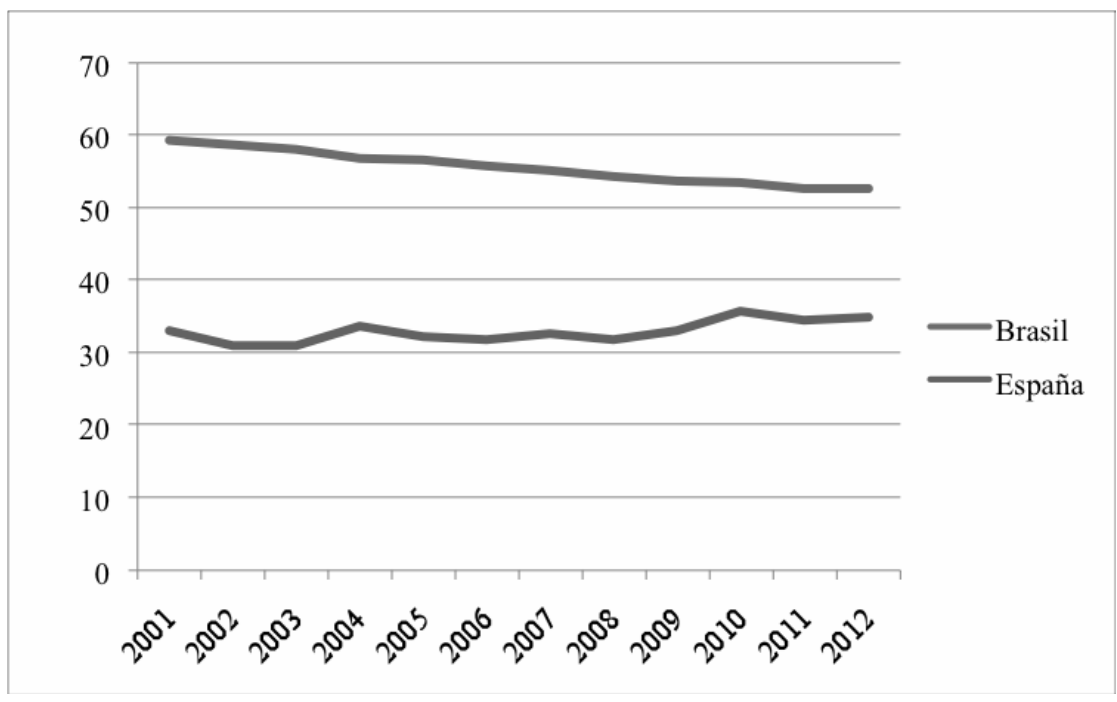

Fuente: elaboración propia a partir de los datos del Banco Mundial (http://datos.bancomundial.org/indicador/SI.POV.GINI)

2) En cuanto a las características sociodemográficas de los participantes. Ambas protestas urbanas se caracterizan por el protagonismo de los jóvenes. Estudiantes universitarios y desempleados en España, y estudiantes universitarios y de secundaria, así como subempleados en Brasil. En el desarrollo de las protestas, mientras en España se puede ver un conjunto muy heterogéneo (interclasista e intergeneracional) donde la diversidad coexiste sin grandes conflictos (la doble alma del movimiento que citábamos con Taibo); en el caso de Brasil se produce una incorporación de clase media urbana conservadora que escinde la protesta en dos movimientos antagónicos de izquierda (minorías, clases populares y clases medias progresistas) y de derecha (clases medias emergentes), donde los segundos adquieren mayor protagonismo en las fases finales. Supone un cambio ideológico de las protestas que "coincidió con una caída abrupta del número de manifestantes. El movimiento que comenzaba apartidario se tornaba antipartidario" (Secco, 2013: 74). Aunque la aversión inicial a siglas de partidos y sindicatos, así como de otros símbolos de movimientos tradicionales, es común en los dos movimientos; en el momento del protagonismo conservador se manifiesta el rechazo al PT y a los partidos de la izquierda política.

3) Esa transfiguración de la protestas en Brasil, hace que en las demandas pierdan peso las cuestiones relacionadas con la calidad de los servicios públi- 
cos y los excesos presupuestarios de la Copa Mundial de Fútbol, así como las cuestiones ambientales (obras hidroeléctricas) y rurales (usurpación de tierras) en contraste con las protestas contra la corrupción política del partido del gobierno. Las protestas, paradójicamente, son aprovechadas tras el impulso inicial de la izquierda por las fuerzas más conservadoras de un país tradicionalmente conservador como es Brasil. Una clase media emergente que está a favor de la reducción de la edad penal, en contra del matrimonio gay, contra los derechos de la mujer y a favor del genocidio de los jóvenes negros y pobres de las perifereias de las ciudades (Sakamoto, 2013: 97).

4) En cuanto a los últimos desarrollos se presentan unas diferencias acentuadas en las dos protestas estudiadas. En España se produce una bifurcación del movimiento (segmentación con concreción de demandas a partir de las mareas de defensa de los servicios públicos y el Estado de Bienestar, por un lado, y marchas y protestas con repertorios de confrontación que reclaman una profunda transformación social, por otro) con importantes elementos en común (tanto de individuos particulares como de denuncias de la situación social). Sin embargo, en Brasil, aunque las Jornadas de Junho han proporcionado un incremento de la capacidad de movilización de movimientos sociales tradicionales de izquierda, se tiene la sensación de que estos movimientos no han sido tan capaces de aglutinar el descontento como lo ha hecho la derecha. En el momento actual (junio de 2015) el movimiento 15M está en vías de codificar sus demandas en una propuesta electoral (Podemos) que se ha convertido en una fuerza política emergente (tercera en votos en las últimas elecciones municipales y autonómicas); mientras las Jornadas de Junho sirven de referencia a una amalgama de movimientos de fuerte carácter conservador (con tintes xenófobos y clasistas) que denuncia tanto la corrupción como las ayudas del Estado a los sectores más desfavorecidos. Las recientes protestas de los meses de marzo de 2015 donde una amalgama de grupos apartidarios de derecha (neoliberales o neocon, Marra, 2015) se moviliza tras su derrota electoral para pedir la destitución de la presidenta del país por procedimientos de dudosa viabilidad -impeachment- o incluso reclamando la intervención militar.

\section{CONCLUSIONES}

Una vez establecidas estas divergencias y convergencias en el ámbito estatal podemos observar que las coyunturas en las que surgen las protestas son muy diferentes, si bien las cuestiones estructurales son comunes (salvando las considerables distancias desde el punto de vista histórico y cultural), en el sentido de estar afectadas por la misma ola neoliberal que afecta al capitalismo global actual. Desde esa perspectiva, las protestas son reacciones de ambas sociedades 
civiles ante la renuncia del Estado a la protección social y a la mejora de las condiciones de vida.

Consideramos que la privación y la frustración de expectativas (Gurr,1974; Smelser, 1989) no es suficiente para explicar ambas movilizaciones, y que se han de atender a otros condicionamientos (marcos de interpretación y oportunidades políticas), pero es de destacar que en este caso se produce una coincidencia entre factores subjetivos y objetivos. No hay lugar aquí para profundizar sobre los aspectos psicológicos motivacionales que impulsan a los individuos hacia la protesta, pero se observa cómo los datos objetivos de la situación socioeconómica (deterioro en España, quiebra de expectativas de mejora en Brasil) se complementan con la percepción subjetiva de la movilidad social intergeneracional. En un estudio reciente (Alaminos, Penalva, Domenech, 2014) a partir de los datos de la Pew Research Center Global Attitudes Project, de 2012, se observan las expectativas de movilidad social intergeneracional de ambos países. En el caso español y brasileño, sus sociedades piensan que viven mejor que las generaciones anteriores (padres); pero hay diferencias en cuanto a la percepción sobre el futuro de la generación descendiente (hijos). Así, en cuanto a las expectativas de movilidad social intergeneracional en España se percibe un notable pesimismo, mientras que en Brasil se observa optimismo.

Desde el punto de vista de los "marcos de interpretación" (Gamson y Meyer, 1999; Tarrow, 2004, McAdam, Tarrow, Tilly, 2005), se contempla la idea de "injusticia" en ambos escenarios y la idea de renovación política y social que

Tabla 1. Percepción de la movilidad social intergeneracional (Brasil y España)

\begin{tabular}{l|c|c}
\hline & Brasil & España \\
\hline $\begin{array}{l}\text { Percepción movilidad social ascendente } \\
\text { (con respecto a la generación anterior) }\end{array}$ & $81,70 \%$ & $71,70 \%$ \\
\hline $\begin{array}{l}\text { Expectativas de movilidad social } \\
\text { ascendente de los } \text { hijos }^{14}\end{array}$ & $59,20 \%$ & $5,50 \%$ \\
\hline
\end{tabular}

Fuente: elaboración propia a partir de Alaminos, Penalva, Domenech, 2014

${ }^{13}$ Suma de porcentajes de respuesta "much better" y "somewhat better" de la pregunta: Compared to your parents when they were the same age as you are now, do you think your own standard of living now is much better, somewhat better, about the same, somewhat worse, or much worse than theirs was?

${ }^{14}$ Suma de porcentajes de las respuestas "very easy" y "somewhat easy" a la pregunta: How easy or difficult is it in our country for a young person to get a better job and to become wealthier than his or her parents were - very easy, somewhat easy, somewhat difficult or very difficult? 
afecte sustancialmente a las estructuras políticas del Estado. El concepto, procedente del constructivismo, es útil para entender las dinámicas de la acción colectiva, en el sentido de los significados compartidos por medio de los cuales la gente tiende a definir la situación. Estos marcos pueden proporcionar a la protesta el diagnóstico, las orientaciones afectivas y cognitivas, así como aspectos identitarios de pertenencia al grupo y de definición de los adversarios políticos. Desde esta perspectiva, en ambos procesos se detectan las oportunidades políticas derivadas de la percepción de sistemas políticos en descomposición, con una importante pérdida de legitimidad de las instituciones; y un sistema político alejado de los consensos alcanzados tras las transiciones democráticas desde las dictaduras. Conviene señalar la coincidencia de las movilizaciones en España en pleno año electoral (municipales y generales) y que las protestas en Brasil tuvieron lugar en el año anterior a las elecciones presidenciales y estatales; movilizaciones que reflejaban el descontento con la socialdemocracia gobernante (PSOE y PT, respectivamente). En el caso brasileño, además, la protesta coincidía con la visualización internacional de los preparativos de la Copa Mundial de Fútbol del año siguiente.

Los ciclos de protestas vienen a expresar el proceso de experimentación, extensión e intensificación que se produce tanto espacialmente como en el tiempo. El concepto, procedente de Tarrow (2004), se entiende aquí como un proceso que con la prolongación de la protesta, propicia la aparición y desarrollo de nuevas organizaciones, consolidan las antiguas y permiten la interacción entre lo nuevo y lo viejo creando una especie de fusión que da lugar a un nuevo marco de interpretación compartido. En España, resulta muy interesante cómo llegan a converger en multitud de protestas individuos (y en ocasiones colectivos) experimentados en largas luchas por la transformación social con personas desencantadas que se inician en la participación política no convencional y que buscan una serie de reformas que les conduzcan a la situación anterior a la crisis. En Brasil, las movilizaciones de Junio de 2013 siguen la estela de luchas emprendidas en más de una década por el mismo MPL, con intentos de convergencia y adaptación al medio urbano de las formas de lucha del MST, y con el mismo carácter anticapitalista (Zibechi, 2014).

Se observa que en esta convergencia, las nuevas incorporaciones a este ciclo renuevan el carácter de los llamados "nuevos movimientos sociales" (Gunder Frank y Fuentes, 1987; Melucci, 1996, De Sousa Santos, 2001). En este sentido se puede ver su fuerte carácter identitario e interclasista, la búsqueda de formas horizontales y comunitarias de organización y autogestión política, la renuncia a los fuertes liderazgos, la desvinculación de las organizaciones políticas tradicionales y la renuncia al poder. 
Aunque en el caso de Brasil, las jornadas de junho han posibilitado la organización y movilización de los sectores más conservadores del país; para los amplios sectores más afectados por las políticas neoliberales, el marco de interpretación creado en ambas sociedades es el de descomposición de lo viejo y construcción de algo nuevo. La importancia de los marcos de interpretación ha sido destacada por multitud de autores, señalando este importante componente cognitivo a la vez que ideológico de definición de la situación que impele a los individuos a la acción colectiva. Estos marcos son condicionados por la tradición cultural e histórica en la acción colectiva y, a su vez, determinan los "repertorios "de la acción colectiva que se desarrolla en un momento determinado. Repertorios que intensifican tanto las estrategias heredadas de búsqueda espectacularidad y simbolismo (batucadas, ambiente carnavalesco, expresividad emocional), de desobediencia pacífica y de ocupación de espacios públicos que estimulan el seguimiento mediático; como nuevas, muy relacionadas con el uso de la redes sociales tecnológicas y que han provocado la producción de numerosos ensayos desde la perspectiva etnográfica (Postill, 2014); en su conexión con la vertiente emocional (Perugorría y Tejerina, 2013) o que han aplicado al estudio de las protestas de España y Brasil nuevos conceptos como la "tecnopolítica" (Toret, 2013) y el "ciberactivismo" (Bentes, 2013; en el prefacio a la obra de Malini y Antoun). Estos estudios incorporan la capacidad de las redes sociales (en tiempo real, con el empleo de imágenes y retórica textual de caracteres reducidos) para incrementar la identificación emocional con el movimiento tanto en los momentos de estallido como en su desarrollo.

El estudio de los movimientos sociales nos permite observar qué es lo que está cambiando en una sociedad en términos de mentalidad, costumbres, y prácticas políticas y sociales. También internacionalmente. El movimiento iniciado en la primavera árabe pasó por la península ibérica para llegar a Londres y Nueva York, Estambul, Sao Paulo, México DF, Santiago de Chile, Hong Kong. Con el proceso de globalización económica y cultural los movimientos no se han podido nunca abordar sin tener en cuenta su interconexión, sus mutuas influencias en el ámbito internacional. En cierto modo, con los desarrollos tecnológicos de la comunicación se asiste a un diálogo más intenso entre movimientos que en anteriores épocas, y que -con sus especificidades nacionalesincluso se podría hablar de una protesta coral contra el capitalismo como forma de organización social que en la actualidad afecta a toda la humanidad. Así es cómo interpreta Žižek (2013) todas estas protestas de los últimos años, señalando que dentro de la diversidad, todas las protestas son reacciones a las diferentes facetas de la globalización capitalista. Un capitalismo internacional que está según indican muchas fuentes en una fase terminal (Harvey, 2014). Realmente 
lo que está en juego es la forma que adoptará algo que no se sabe que será (Wallerstein, 2004, Bauman, 2012) y no cabe duda de que los movimientos sociales (con notables limitaciones programáticas y organizativas) están ensayando con sus prácticas y sus propuestas el surgimiento de algo nuevo a nivel global que debe estar relacionado con los principios de libertad y la igualdad. Es algo que se discutió en las ediciones del Foro Social Mundial -cuyas primeras ediciones se celebraron precisamente en Brasil, Porto Alegre- por parte de colectivos sociales de todo el mundo, y que ahora adquiere plena vigencia.

\section{BIBLIOGRAFÍA}

Alaminos, A. y Penalva C. (2013). "España: de los impactos de la crisis a las movilizaciones de protesta". Ecuador Debate. Agosto. 93-118

Alaminos, A. Penalva, C. y Domenech, Y. (2014b). "A mobilidade social intergeracional subjetiva e as expectativas em relação ao futuro (Brasil, Rússia, Índia em comparação com Espanha, Itália e Grã-Bretanha), en $2^{\circ}$ Encontro Internacional de Política Social, UFES, (Brasil), August 4th -7th 2014.

Auditoria Cidadã da Dívida. auditoriacidada.org.br, acesso em agosto de 2014.

Avritzer, L. (2007). "Sociedade civil, instituições participativas e representação: da autorização à legitimidade da ação", DADOS, Revista de Ciências Sociais, Rio de Janeiro, vol. 50, n.3, 443-464.

Bauman, Z. (2012). "Times of Interregnum". En: Ethics and Global Politics, Vol. 5, núm. $1,49-56$.

Baumgarten, B. (2013). "Geracao a Rasca and beyond: Mobilizations in Portugal after 12 March 2011", Current Sociology, 61(4) 457-473.

Bravo, M. I. S. (2012). "Desafios do controle social na atualidade". Serviço Social \& Sociedade, vol. 109, 126-150.

Bentes, I. (2013). "Nós somos a rede social" prefácio en F. Malini y H. Antoun en @internet e \#rua ciberativismo e mobilização nas redes sociais. Porto-Alegre. Sulina, 9-16

Castells, M. (2012), Redes de indignación y esperanza. Madrid. Alianza Editorial.

Clementi, F. y Schettino, F. (2013). "Polarização de Renda no Brasil, de 2001 a 2011: uma análise distribucional usando os dados PNAD", Revista Praia Vermelha, Rio de Janeiro, v.23, n.1, p.33-68, Jan/Jun 2013.

Dagnino, E. (2004), "Sociedade civil, participação e cidadania: de que estamos falando?", en D. Mato (coord.) Políticas de ciudadanía y sociedad civil en tiempos de globalización. Caracas, FASES, Universidad Central de Venezuela.

Della Porta, Donatella y Tarrow, Sidney (2005) Transnational protest and Global Activism. People, Passions and Power, Lanham, MD, Rowman \& Littlefield.

De Sousa Santos, Boaventura (2001), "Los nuevos movimientos sociales", OSAL, No. 5. $177-184$

DIEESE (2012), A situação do trabalho no Brasil na primeira década dos anos 2000. São Paulo, DIEESE. Disponíble en: http://www.dieese.org.br/livro/2012/livroSituacao TrabalhoBrasil.pdf 
DIEESE (2014), Os negros nos mercados de trabalho metroplitanos. Pesquisa de Emprego e Desemprego, 2014. São Paulo. DIESSE 2014. Disponible en: https://www. dieese.org. br/analiseped/2013/2013pednegrosmet.pdf

Domenech, X. (2014). Hegemonías. Crisis, movimientos de resistencia y procesos políticos (2010-2013), Madrid. Akal.

Esping-Andersen, G. (1991). "As três economias políticas do Welfare-State". Lua Nova, São Paulo: Marco Zero e CEDEC, n.24, p.85-116, set/1991.

Gamson, W. y Meyer, D.S. (1999). "Marcos interpretativos de la oportunidad política", en D. McAdam, J. D. McCarthy y M. N. Zald (eds.), Movimientos sociales: perspectivas comparadas. Madrid. Ediciones Itsmo, 389-412

Gohn, M. G. (2013). Sociologia dos movimentos sociais, São Paulo. Cortez. Coleção Questões da Nossa Época, v.47.

Gurr, Ted (1971). Why Men Rebel? Princeton, Princeton University Press.

Harvey, D. (2014). Diecisiete contradicciones y fin del capitalismo, Madrid, Traficantes de sueños.

Hughes, N, (2011), "Young People Took to the Streets and all of a Sudden all of the Political Parties Got Old': The 15M Movement in Spain", Social Movement Studies: Journal of Social, Cultural and Political Protest, Volume 10, Issue 4, 407-413.

Instituto Brasileiro de Geografia e Estatística (IBGE) (2010). Censo Demográfico 2010/T rabalho e Rendimento: resultado da amostra. Rio de Janeiro. Ministério do Planejamento, Orçamento e Gestão.

Marra, A. (2015), “¿Quiénes están detrás de las protestas en Brasil contra Dilma Rousseff?", en Publico.es, 17/3/2015, Disponible en http://www.publico.es/internacional/quienes-detras-protestas-brasil-dilma.html

McAdam, Doug, Tarrow, Sindney y Tilly, Charles (2005), Dinámica de la contienda política, Barcelona, Hacer S.L.

Perugorría, I. y Tejerina, B. (2013) "Politics of the encounter: Cognition, emotions, and networks in the Spanish 15M", Current Sociology 0(0) $1-19$.

Postill, John (2014), "Democracy in an age of viral reality: A media epidemiography of Spain's indignados movement", Ethnography, Vol. 15(1) 51-69.

Sakamoto, L. (2013), "Em São Paulo, o Facebook e o Twitter foram às ruas", en C. Vainer (et. al.), Cidades rebeldes. Passe livre e manifestações que tomaram as ruas do Brasil. São Paulo. Boitempo Editorial. pp. 95-100.

Radis (2014), "Plebiscito Popular: 7,4 milhões pela reforma política", RADIS, n.146, Nov. 2014.

Smelser, Neil J. (1989). Teoría del comportamiento colectivo. México, Fondo de Cultura Económica.

Secco, L. (2013), "As Jornadas de Junho" en C. Vainer (et. al.), Cidades rebeldes. Passe livre e manifestações que tomaram as ruas do Brasil. São Paulo. Boitempo Editorial. pp. 71-78.

Taibo, C. (2011), Nada será como antes, Madrid. Los Libros de la Catarata.

Taibo, C. (coord.) (2012) ¡Espabilemos! Argumentos desde el 15M, Madrid. Libros de la Catarata. 
Tarrow, Sidney (2004), El poder en movimiento. Los movimientos sociales, la acción colectiva y la política, Madrid, Alianza Editorial.

Toret J. (2013), Tecnopolitica: el poder de las multitudes conectadas. Barcelona. Universitat Oberta de Catalunya (UOC).

Tatagiba, L. (2012). "Os conselhos gestores de políticas públicas sob o crivo da política: balanços e perspectivas", Serviço Social \& Sociedade, vol. 109, 68-92.

UOL (2014), "UOL Eleições 2014", Disponível: http://www.uol.com.br/eleicoes.

Wallerstein, I. (2004), "Las nuevas rebeliones antisistémicas"; ¿Un movimiento de movimientos? Contrahistorias. La otra mirada de Clío. Vol. 1, num. 1, sep-feb, 77-86.

Zibechi, R. (2014). Descolonizar la rebeldía. (Des)colonialismo del pensamiento crítico y de las prácticas emancipatorias. Málaga. Baladre/Zambra.

Žižek, S. (2013). "Problemas no Paraíso", en C. Vainer (et. al.), Cidades rebeldes. Passe livre e manifestações que tomaram as ruas do Brasil. São Paulo. Boitempo Editorial. pp. 101-108.

CLEMENTE PENALVA VERDÚ Profesor Titular, Departamento de Sociología II, e investigador del Instituto Interuniversitario de Desarrollo Social y Paz. Universidad de Alicante, España. Líneas de investigación: capital social y movilización social; redes de apoyo mutuo y redes para la acción política; la dimensión económica de la confianza social: Últimas publicaciones: "Antiausteridad y protesta en el contexto de la crisis económica y política en España e Italia" (con L. Raffini y A. Alaminos); "España: de los impactos de la crisis a las movilizaciones de protesta", Ecuador Debate, 2013 (con A. Alaminos);

ANA TARGINA RODRIGUES FERRAZ Professora associada do Departamento de Serviço Social e do Programa de Pós-Graduação em Política Social da Universidade Federal do Espírito Santo, Brasil. Linhas de investigação: movimentos sociais, democracia, canais de participação popular e políticas sociais. Últimas publicações: "Políticas sociais na contemporaneidade: o fim do caminho?", revista Textos e Contextos (Porto Alegre), 2014; "Brazil: spring between the teeth", revista Critical and Radical Social Work, 2013

Recibido: 09/09/2015

Aceptado: 30/12/2015 